\title{
Upward spinal coning: impaction of occult spinal tumours following relief of hydrocephalus
}

\author{
RASHID JOOMA, RICHARD D HAYWARD \\ From the Departments of Neurosurgery, The Hospital for Sick Children, and The National Hospital for \\ Nervous Diseases, Queen Square, London, UK
}

\begin{abstract}
SUMMARY Neurological deterioration sometimes follows removal of cerebro-spinal fluid by lumbar puncture below a spinal block caused by a tumour. Experience has shown that similar impaction of a spinal intradural tumour may occur following the draining of hydrocephalus. We have used the term "upward spinal coning" to describe this and report four cases in which impaction of an intradural metastasis occurred when the obstructive hydrocephalus caused by the primary intracranial tumour was relieved. The possible mechanisms are discussed. Awareness of the syndrome is important for early diagnosis and treatment and motor power should be assessed regularly following the relief of hydrocephalus caused by a tumour known to seed in the spinal subarachnoid space.
\end{abstract}

The herniation of neural elements between the cranial compartments in response to increased pressure in one compartment is a well-defined concept in neurosurgery. Cushing ${ }^{1}$ drew attention to the "dislocation downward into the foramen magnum" when discussing the consequences of increased intracranial tension of the medulla, while two years later Collier ${ }^{2}$ used the term "pressure cone" to describe cerebellar tonsillar herniation. In patients with intracranial mass lesions, significant pressure differentials may exist between the cranial and the spinal compartments ${ }^{3}$ and further lowering of the lumbar pressure by a spinal CSF tap may precipitate or aggravate herniation through the tentorial hiatus and the foramen magnum. Lumbar puncture can also initiate neurological deterioration when CSF is withdrawn below a spinal block caused by an extra-medullary tumour; 4 the lowering of the pressure below causes impaction of the tumour and the "pressure cone" compresses and compromises the adjacent neural structures of the spinal cord. Recent experience of four cases has shown how a previously unrecognised upward form of coning can occur following the relief of hydrocephalus. In these examples, the sudden reduction of intracranial pressure

Address for reprint requests: Mr RD Hayward, Department of Neurosurgery, National Hospital for Nervous Diseases, Queen Square, London WC1N 3BG, UK.

Received 12 July 1983 and in revised form 28 October 1983. Accepted 5 November 1983 has produced symptoms and signs referable to previously undeclared spinal tumours.

\section{Case reports}

Case 1

This 8-year-old girl presented with a five-month history of intermittent headache and vomiting and, latterly, increasing lethargy. Examination revealed bilateral papilloedema and symmetrical hypertonicity of her legs but with no weakness. Computed tomography (CT) demonstrated hydrocephalus due to a large pineal region tumour with intraventricular metastases in the trigone of the left lateral ventricle, the temporal horn of the right ventricle and the fourth ventricle. On a presumptive diagnosis of germinoma or pineoblastoma, dexamethasone was given and a ventriculo-peritoneal shunt inserted as a prelude to radiotherapy. During the first twenty-four hours after operation, she was very drowsy but on the second day she was sufficiently alert to reveal a moderate weakness of the left leg. The following day she had become paraplegic with an incomplete sensory level at T4. A myelogram (fig 1) disclosed multiple intradural extra-medullary filling defects, the largest one being in the upper dorsal region and she was urgently referred for radiotherapy. However, her general condition continued to decline during the six days she received treatment. She died fourteen days after the insertion of the shunt and necropsy confirmed spinal subarachnoid metastases from a pineal tumour (fig 2). Histology showed a pineoblastoma.

Case 2

This 10-year-old boy was seen because of a three-month history of headache, vomiting and, more recently, double 
vision and difficulty in walking. He was noted to have papilloedema, a right VI nerve palsy and truncal ataxia. The CT scan showed hydrocephalus due to a fourth ventricular mass. A sub-occipital craniectomy in the sitting position was performed with a near-total excision of a tumour diagnosed, on subsequent histological examination, as a medulloblastoma. His immediate recovery from surgery was excellent but on the second post-operative day, he developed urinary retention and required catheterisation. He had great difficulty mobilising and at first this was attributed to his ataxia. However, a week later, when abdominal distension was noted without any obvious local cause, the possibility of spinal metastases was raised. A myelogram confirmed the presence of multiple spinal tumours (fig 3 ) and in retrospect it was appreciated that the large metastasis at the conus had been responsible for his post-operative deterioration. Radiotherapy was commenced immediately.

Case 3

Two months prior to admission this 20-year-old man

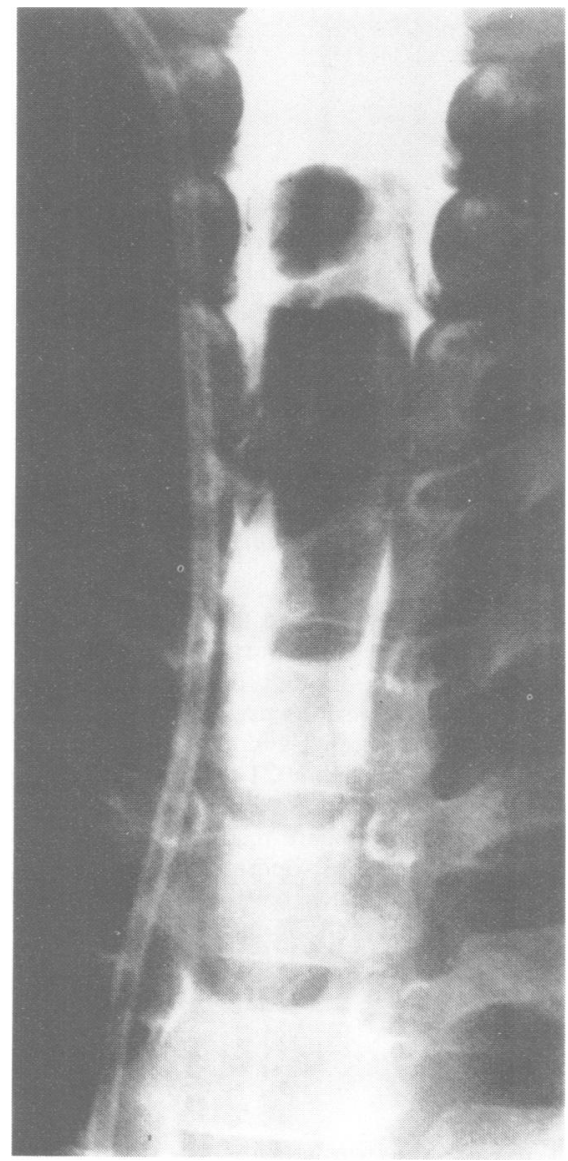

Fig 1 Case 1. AP projection of myelogram. Two intradural masses are noted in the upper dorsal region. 


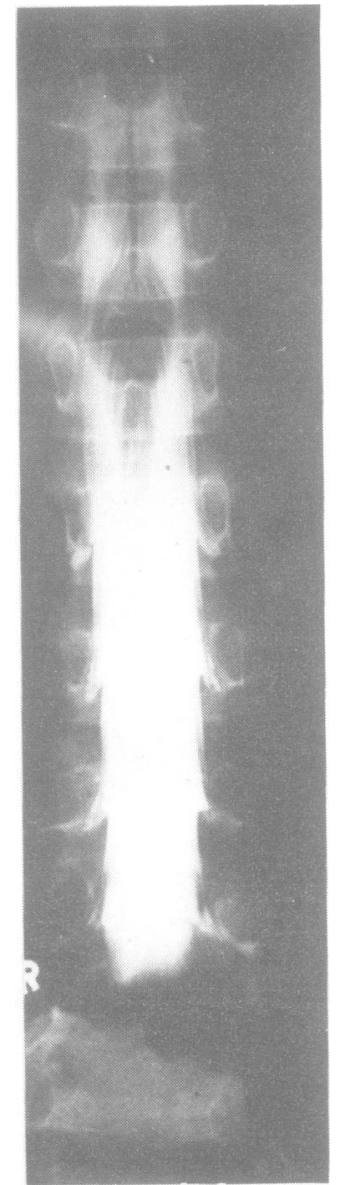

Fig 3 Case 2. Myelogram with an intradural tumour just above the conus at D12.

extensor but there was no evidence of increased tone or weakness in the legs. A CT scan showed moderate hydrocephalus due to a small enhancing fourth ventricular tumour and a diagnosis of medulloblastoma probably complicated by spinal secondaries was made. A posterior fossa exploration with a near total removal of the tumour (confirmed histologically as a medulloblastoma) was performed and the patient awoke from his anaesthetic without additional deficit. However, by the following day he had developed a profound paraparesis with a sensory level at T10. Myelography showed a block at T12 (fig 4). He underwent a decompressive laminectomy and partial removal of a large medulloblastoma metastasis followed by craniospinal irradiation and chemotherapy. At one year's follow-up he could walk only with splints and a check myelogram showed no residual tumour.

\section{Case 4}

At the age of 13 years, this girl underwent a near total excision of a medulloblastoma followed by radiotherapy.

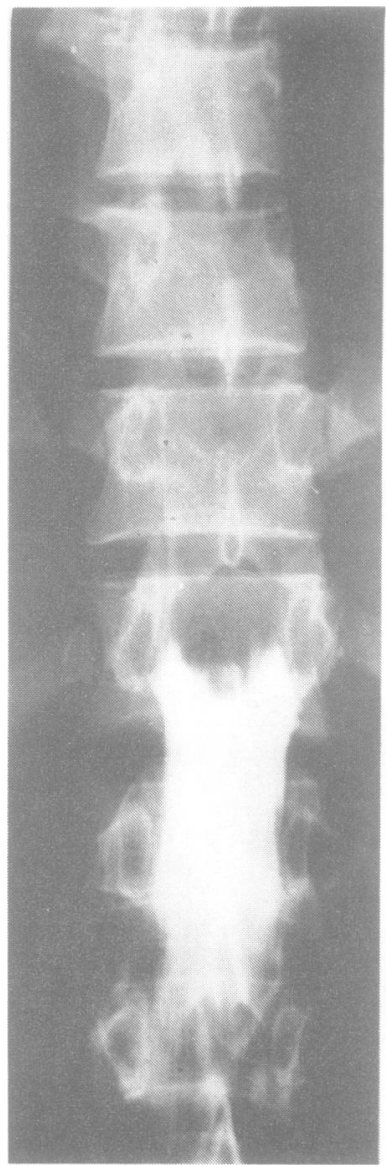

Fig 4 Case 3. At myelography, a complete block at D12 is seen due to an intradural tumour.

She did well for five years and then returned with clinical and CT scan evidence of a fourth ventricular recurrence, associated with moderate hydrocephalus. At reexploration dense adhesions prevented adequate tumour resection and after a few days' relief, the headache and vomiting returned. A further CT scan confirmed the persistence of hydrocephalus and so a ventriculoperitoneal shunt was inserted with immediate resolution of her symptoms. However, on the third post-operative day (when her mobilisation was commenced) it became obvious that she was having difficulties in walking and examination showed a mild paraparesis and hyper-reflexia. A myelogram demonstrated multiple spinal tumours (fig 5 ) and she was referred for radiotherapy.

\section{Discussion}

Based on post-mortem studies, Meyer ${ }^{5}$ in 1920 drew attention to uncal herniation into the tentorial hiatus in patients with expanding cerebral lesions as the 


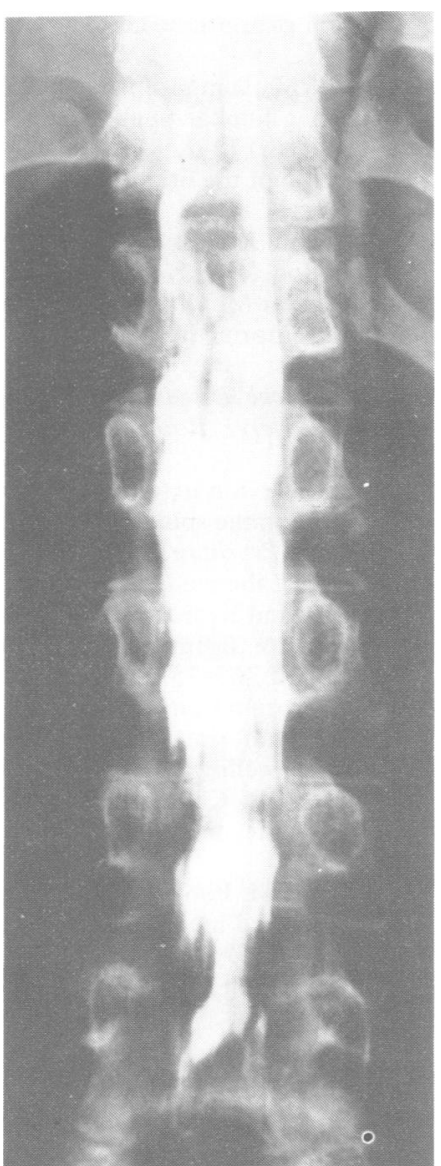

Fig 5 Case 4. Myelogram (using water-soluble medium) reveals multiple filling defects caused by extra-medullary metastases from the medulloblastoma.

cause of brainstem failure. It soon became apparent that a lumbar puncture could be disastrous in cases of space-occupying lesions by increasing the pressure gradients that favour cerebral herniation. An "upward cone" is also recognised intracranially when an expanding posterior fossa mass causes herniation into the tentorial notch with brainstem compression and this process can be encouraged by withdrawing CSF from the lateral ventricles. ${ }^{67}$ Similar mechanical considerations may be thought to apply to the acute cord dysfunction that occurs when a CSF shift by lumbar puncture or relief of hydrocephalus causes a spinal extra-medullary tumour to impact against the neural elements. We have termed this process "spinal coning" and consider that it can be either downward following a spinal tap or, as in the four cases described above, upward when the ventricles have been drained.
Elsberg ${ }^{8}$ explained aggravation of the neurological disturbance of an extra-medullary spinal growth after lumbar puncture as being due to removal of the buffer of spinal fluid between the tumour and the spinal cord, allowing one to fall against the other. The role of movement in the aetiology is supported by personal observations made by one of the authors of this communication (RDH). At a lumbar laminectomy for a large neurofibroma, the dura was opened and the tumour could be seen through the arachnoid occupying the upper half of the exposure. When the arachnoid below the tumour was incised there was, along with a brisk flow of CSF, a downward movement of the tumour by about $1.5 \mathrm{cms}$. A similar event occurred during a laminectomy for a dorsal neurofibroma but not surprisingly the movement observed was less: $2-3 \mathrm{~mm}$.

While alteration of the CSF pressure gradient across the spinal tumour would appear to be the most important factor in its shift and impaction, movement of the cord itself may also contribute. Although anchored by the dentate ligament, the cord has a small range of mobility expressed during flexion-extension movements of the head and spine ${ }^{9}$ and deep breathing. ${ }^{8}$ Hydrocephalus causes some caudal movement of the brainstem and cord ${ }^{10-12}$ and reduction of the ventricular size by draining could conceivably result in a small upward movement of the cord. This, in turn, could lead to venous obstruction of both the tumour and the segment of the cord to which it is affixed causing swelling of both structures and increasing the degree of compression.

The four patients we have described all presented with obstructive hydrocephalus due to tumours with a known predilection to seed within the spinal subarachnoid space, although only one (Case 3 ) had any symptoms referable to the spine at presentation. However, following relief of the hydrocephalus - by excision of tumour in Cases 2 and 3 and by a ventriculoperitoneal shunt in Cases 1 and 4-the occult spinal metastases became clinically manifest.

Other explanations for the sudden deterioration of these patients such as coincidence, failure to appreciate clinical evidence of cord compression pre-operatively and accidents that occurred during surgery are all difficult to support. In Cases 2 and 4 there was a slowness to recognise the symptoms and signs of cord compression but this was after surgery in patients whose consciousness level before operation (as in Cases 1 and 3) had allowed a detailed neurological examination. The different operations performed (two posterior fossa explorations in the sitting position and two shunt insertions with the patients supine) make a single local surgical event unlikely. Certainly, intradural spinal tumours can present acutely but this is most unusual and the 
striking coincidence in all four cases seems to be the onset of cord compression within at the most three days (Case 4) of their operation.

A variety of cerebral and cerebellar tumours can be complicated by spinal metastases ${ }^{13}$ but the most important are medulloblastomas, ${ }^{14} 15$ fourth ventricle ependymomas, ${ }^{1617}$ and pineal tumours; ${ }^{1819}$ as these neoplasms also usually cause obstructive hydrocephalus, the syndrome of upward spinal coning following relief of hydrocephalus will most often be associated with them. Although it is of obvious importance to recognise this syndrome, it must remain a rare complication and the lack of symptoms indicating spinal disturbance in three out of four cases points to the difficulties of making a preoperative clinical diagnosis. The multiplicity of the spinal secondaries in our cases appeared to contraindicate direct surgery and only Case 3 underwent a decompression, the rest being referred for primary irradiation. Case 4 has made a good recovery from this approach.

The syndrome of upward spinal coning is caused by impaction of a spinal secondary when the obstructive hydrocephalus associated with the primary intracranial tumour is relieved. There will not usually be any clinical clues enabling prediction of this complication before cranial surgery but awareness of the syndrome and clinical vigilance-with repeated testing of motor power-after relieving hydrocephalus due to third and fourth ventricular tumours should result in early recognition and treatment.

\section{References}

' Cushing H. Some experimental and clinical observations concerning states of increased intracranial tension. Am J Med Sci 1902;124:375-400.

${ }^{2}$ Collier $\mathrm{J}$. The false localising signs of intracranial tumor. Brain 1904;27:490-508.

${ }^{3}$ Kaufman GE, Clark K. Continuous simultaneous monitoring of intraventricular and cervical subarachnoid cerebrospinal fluid pressure to indicate develop- ment of cerebral or tonsillar herniation. J Neurosurg 1970;33:145-50.

4 Eaton LM, Craig WM. Tumor of the spinal cord: sudden paralysis following lumbar puncture. Proc Staff Meet Mayo Clin 1940;15:170-2.

5 Meyer A. Herniation of the brain. Arch Neurol Psychiat 1920;4:387-400.

- Cuneo RA, Caronna JJ, Pitts L et al. Upward transtentorial herniation. Arch Neurol 1979;36:618-23.

${ }^{7}$ Eisenberg HM, Sarwar M. Ventriculographic features of ascending transtentorial herniation. Acta Neurochir 1978;42:225-8.

${ }^{8}$ Elseberg CA. Surgical Diseases of the Spinal Cord, Membranes and Nerve Roots. London: HK Lewis, $1942 ; 598$.

${ }^{9}$ Reid JD. Effects of flexion-extension movements of the head and spine upon the spinal cord and nerve roots. $J$ Neurol Neurosurg Psychiatry 1960;23:214-21.

${ }^{10}$ Emery JL. Kinking of the medulla in children with acute cerebral oedema and hydrocephalus and its relationship to the dentate ligament. J Neurol Neurosurg Psychiatry 1967;30:267-75.

"Howell DA. Upper brain stem compression and foraminal impaction with intracranial space-occupying lesions and brain swelling. Brain 1959;82:525-50.

12 Johnson RT, Yates PO. Clinico-pathological aspects of pressure change at the tentorium. Acta Radiol 1956;46:242-9.

${ }^{13}$ Wood EH, Taveras JM, Pool JL. Myelographic demonstration of spinal cord metastases from primary brain tumours. AJR 1953;69:221-30.

${ }_{14}$ Polmeteer FE, Kernohan JW. Meningeal gliomatosis: a study of forty-two cases. Arch Neurol $P$ 1947;57:593-616.

is Strang RR. Intraspinal metastases from medulloblastomas of the posterior fossa. Med J Aust 1962;2:507-10.

${ }^{16}$ Bryan P. CSF seeding of intracranial tumours: a study of 96 cases. Clin Radiol 1974;25:355-60.

${ }^{17}$ Renauding JW, Ditullio MV, Brown WJ. Seeding of intracranial ependymomas in children. Childs Brain 1979;5:408-12.

${ }^{18}$ Fowler FD, Alexander E, Davis CH. Pinealoma with metastases in the central nervous system. A rationale of treatment. J Neurosurg 1956;13:271-88.

19 Jooma R, Kendall BE. Diagnosis and management of pineal tumours. $J$ Neurosurg 1983;58:654-65. 